$$
\begin{aligned}
& N 69.32485 \\
& \text { CR. } 103656
\end{aligned}
$$

ELECTROSTATIC POTENTIAL DISTRIBUTION

OF THE SUNLIT LUNAR SURFACE

April 23, 1969

\title{
Bellcomm, Inc.
}

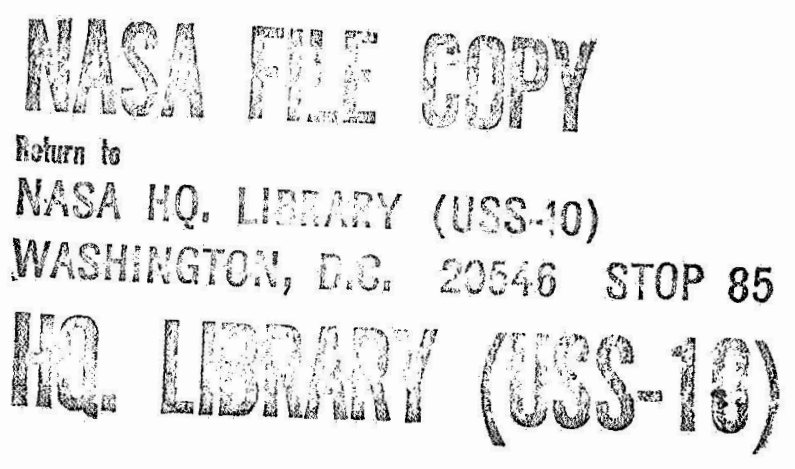


BELLCOMM, INC.

TR-69-710-2

washington, D. C.

\section{ELECTROSTATIC POTENTIAL DISTRIBUTION \\ OF THE SUNLIT LUNAR SURFACE}

April 23, 1969

Prepared by:

J. L. Blank

W. D. Grobman

Work performed for Manned Space Flight, National Aeronautics and Space Administration under contract NASW-417. 
BELLCOMM, INC.

TABLE OF CONTENTS

$\underline{\text { Page }}$

ABSTRACT

1. INTRODUCTION

1

2. CURRENT SOURCES AND THEIR RELATION TO THE LUNAR POTENTIAL

1

3. SOLAR WIND FLUX

3

4. PHOTOELECTRON FLUX 6

5. POTENTIAL DISTRIBUTION 8

6. CONCLUSIONS 11

REFERENCES

APPENDIX: Conduction Currents in the Photoelectron Layer and the Lunar Crust

FIGURE TITLES 


\section{BELLCOMM, INC.}

\section{$\underline{\text { ABSTRACT }}$}

The steady state lunar surface potential distribution is determined by the condition that the net current to a small surface area vanish, where the dominant currents are due to photoemission of electrons and collection of solar wind particles. The potential determined in this manner is insensitive to the detailed structure of the solar spectrum and depends parametrically on the photoemissive properties of the lunar surface. For a work function of 5.0 volts and quantum yield of 0.01 , the electrostatic potential during solar minimum decreases from 3.0 volts at the subsolar point to less than a volt near the limb. Plausible ranges for the lunar quantum yield and work function are 0.001 to 0.1 and 4 to 6 volts, respectively, which correspond to a range of potentials at the subsolar point from 0.6 to 10.2 volts. These values assume a solar wind electron number density and temperature of $5 \mathrm{~cm}^{-3}$ and $10^{5} \circ \mathrm{K}$, respectively. The results indicate that corrections to the ion energies measured by the ALSEP Suprathermal Ion Detector Experiment (SIDE) will be unimportant at energies above a few electron volts. 
BELLCOMM, INC.

\section{INTRODUCTION}

The electrostatic potential of the sunlit lunar hemisphere is a central factor in understanding the plasma environment adjacent to the lunar surface. Knowledge of this potential is valuable for interpreting the results of the ALSEP Suprathermal Ion Detector Experiment (SIDE), which measures the energy distribution of charged particles impacting the lunar surface.

The equilibrium potential distribution of the lunar surface is attained when the net current to an arbitrary surface area vanishes. Previous studies (Opik and Singer, 1960 ; Öpik, 1962; Heffner, 1965) have correctly ascribed the dominant currents to photoelectrons from the surface and charged particles from the solar wind. However these works treat the current processes with simplified models leading to values of the sunlit side lunar potential which are an order of magnitude larger than those given by our present calculation. Furthermore, the potential is calculated by assuming a global current balance which necessarily leads to a constant potential on the sunlit hemisphere. A proper formulation requires a local current balance, and results in a potential which varies over the surface.

An exact calculation of the potential is prohibitively difficult at present, but it is demonstrated that the potential distribution is insensitive to a number of simplifying approximations which render the problem tractable and illustrate the parametric dependence of the potential upon the relevant properties of the lunar surface, the solar spectrum, and the solar wind. In particular, since the calculation of the photoelectron flux involves a convolution of the solar spectrum and the photoemission spectrum of the lunar surface, the detailed structures of these spectra are unimportant and they may be approximated by appropriate smooth functions. The flux from the solar wind is calculated by noting that the electron thermal speed is the largest velocity characteristic of solar wind particle motion, and therefore the moon behaves in a manner similar to that of an electrostatic probe. The solar wind particle flux is then largely due to the random electron flux to the lunar surface.

\section{CURRENT SOURCES AND THEIR RELATION TO THE LUNAR POTENTIAL}

The energy sources capable of producing significant currents at the sunlit lunar surface can be shown to be the solar electromagnetic spectrum and the solar wind. Solar 
photons produce photoelectrons at the lunar surface and solar wind particles are collected there.

The order of magnitude of the flux due to photoelectron production is the product of the quantum yield of the lunar surface and the solar flux of photons with energy above the surface work function. For any reasonable values of the quantum yield and work function, the photoelectron flux is orders of magnitude greater than any other possible charge flux. This is verified below and in section 5. Thus when balance between all fluxes is reached, only a small fraction of the emitted photoelectrons can excape, which implies that the sunlit lunar surface attains a positive electrostatic potential. Also, one can conclude that the escaping photoelectron flux $J_{p}$ and the potential distribution of the sunlit hemisphere $\Phi$ are controlled by the magnitude of the other fluxes present.

An estimate of the net flux from the solar wind is $J_{s}=o\left(n_{0} v_{t}\right)$, where $n_{0}$ is the undisturbed solar wind electron number density and $v_{t}$ is the electron thermal speed. Since $v_{t}>$ $\mathrm{V}$, the bulk solar wind velocity, the proton contribution to $\mathrm{J}_{\mathrm{s}}$ is small (although it is not negligible). However, secondary currents caused by the protons impacting the surface are negligible because the probabilities for these processes are small (Hagstrum, 1954; 1960).

All other known energy sources are incapable of producing charge fluxes comparable to that from the solar wind. The flux from a steady state lunar atmosphere or ionosphere is governed by the rate of production of atmospheric particles, e.g., through outgassing, charge exchange with the solar wind, and photoionization, and this rate is too low to be significant (Edwards and Borst, 1958; Weil and Barasch, 1963). Similarly, processes involving cosmic rays and meteoroids are unimportant.

Additional currents result from conduction through the moon due to the existence of potential gradients and from transport processes in the lunar atmosphere, the latter occurring predominantly in a photoelectron cloud above the sunlit surface. It is demonstrated in the Appendix that the lunar surface and the photoelectron cloud are too resistive to discharge potential differences on the lunar surface, and therefore that these currents may be neglected. 


\section{SOLAR WIND FLUX}

An exact calculation of the charged particle flux from the solar wind is difficult. However, the problem is amenable to a simple approximate solution which is sufficiently accurate for our purposes if we assume that any lunar magnetic fields are too small to significantly alter the solar wind particle distribution functions on the sunlit side of the moon. In this case the moon behaves like an emitting electrostatic probe in the absence of a magnetic field.

At the moon, the solar wind proton and electron number densities are $n_{0} \sim 5 \mathrm{~cm}^{-3}$, and the wind has a bulk velocity $\mathrm{V} \sim 400 \mathrm{~km} / \mathrm{sec}$. A representative value for the proton temperature is $5 \times 10^{4}{ }^{\circ} \mathrm{K}$ (Hundhausen et al., 1967), while the electron temperature is $\mathrm{T} \sim 10^{5}{ }^{\circ} \mathrm{K}$ (Montgomery et al., 1968). Thus the protons have a directed kinetic energy $\frac{1}{2} \mathrm{MV}^{2} \sim 1 \mathrm{KeV}$, which is much greater than their thermal energy.

$$
\text { If we assume }|\mathrm{e} \Phi|<\frac{1}{2} \mathrm{MV}^{2} \text {, where } \Phi \text { is the electro- }
$$

static potential of the lunar surface, then the electrostatic force on the protons due to the presence of the moon causes a negligible perturbation on the proton trajectories. The calculation of the proton flux to the moon therefore becomes trivial. We assume that only those protons whose unperturbed orbits intercept the lunar surface are absorbed. There exists supporting evidence for this assumption (Colburn et al., 1967; Ness et al., 1967). This absorption does not result in a significant production of electrons (Hagstrum, 1954; 1960). Since the streaming velocity is much greater than the proton thermal velocity, the solar wind proton flux to the lunar surface is

$$
\mathrm{J}_{+}=\mathrm{n}_{\mathrm{O}} \mathrm{V} \cos \alpha
$$

where $\cos \alpha=\underline{n} \cdot \underline{V} /|\underline{V}|$, and $\underline{n}$ is an inward pointing normal to the surface.

The solar wind electrons have kinetic energies on the order of $10 \mathrm{eV}$ and a thermal speed, $v_{t}=(2 \mathrm{kT} / \mathrm{m})^{1 / 2} \sim 2 \times 10^{3}$ $\mathrm{km} / \mathrm{sec}$, much greater than their streaming speed, $V \sim 400 \mathrm{~km} / \mathrm{sec}$. 
BELLCOMM, INC. $\quad-4-$

Thus on the sunlit side of the moon, the solar wind electrons appear to be thermalized with a small drift velocity. Solar wind electrons are not present adjacent to the dark side where a plasma void is created due to the absorption of solar wind protons at the sunlit side and the inability of proton diffusion, being a thermally driven process, to fill the void. Thus only about half the lunar surface is available for collection of solar wind electrons.

There are several length scales which characterize the plasma environment on the sunlit hemisphere. From Poisson's equation it is readily established that the length scale governing the spatial variation of the electrostatic potential

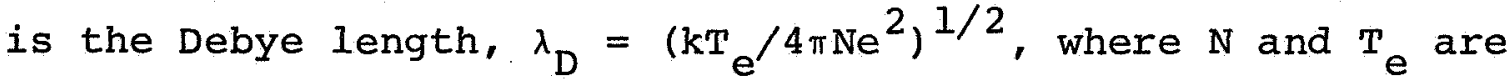
the electron density and temperature, respectively. Most of the decrease to zero of the potential and charge density occurs within a distance of order $\lambda_{D}$ from the surface. In this region there is a photoelectron cloud or sheath which shields the lunar surface charge from the oncoming solar wind. An upper bound for $\lambda_{D}$ is obtained by basing. $\lambda_{D}$ on the solar wind electron density, which gives $\lambda_{D}<10 \mathrm{~m}$ in the lunar electrostatic sheath.

The electron cyclotron radius is $r_{b}=m_{t} / e B \sim 5 \mathrm{~km}$ for typical solar wind parameters. Thus the inequality $\lambda_{D} \ll r_{b}$ is satisfied, and effects of the interplanetary magnetic field are weak and may be neglected. In addition, the inequality $\lambda_{\mathrm{D}} \ll \mathrm{R}$, the lunar radius, permits us to neglect the curvature of the lunar surface, and therefore to treat the moon as locally plane. Since the drift velocity $v<v_{t}$ ' and the Debye length is much less than the electron mean free path, the situation is analogous to the thin sheath approximation in collisionless probe theory (see e.g., Chen, 1965).

Since the lunar potential is positive, there is no repulsion of solar wind electrons, and a good approximation of the electron flux to the moon is the flux entering a surface located a distance $\lambda_{D}$ above the sunlit lunar surface. This flux is insensitive to the lunar potential as long as $e \Phi / \mathrm{MV}^{2} \ll 1$, and is the counterpart of electron saturation in electrostatic probe theory. A locally plane surface element of area ds with inward pointing normal $n$ is used to compute the flux. Then the flux of solar wind electrons to the area ds as a function of $\cos \alpha$ is 


$$
J_{-}(\cos \alpha)=\int_{\underline{n} \cdot \underline{v}>0} d^{3} v(\underline{n} \cdot \underline{v}) f(\underline{v})
$$

where $f(v)$ is the solar wind electron distribution function, $v$ the electron velocity, and the integration is performed over the half-space $\underline{n} \cdot \underline{v}>0$. Employing our thin sheath approximation and assuming the undisturbed solar wind electron distribution function to be Maxwellian,

$$
f=\frac{n_{0}}{(2 \pi k T / m)^{3 / 2}} \exp \left[-\frac{m(\underline{v}-\underline{v})^{2}}{2 k T}\right], \underline{n} \cdot \underline{v}>0
$$

we arrive at

$$
\begin{aligned}
& J_{-}(\cos \alpha)=\frac{n_{0} v_{t}}{2 \sqrt{\pi}}\left\{\exp \left(-u^{2} \cos ^{2} \alpha\right)\right. \\
& +\sqrt{\pi} u \cos \alpha[1+\operatorname{erf}(u \cos \alpha)]\}
\end{aligned}
$$

where $u=v / v_{t}$.

To obtain the net solar wind charge flux we combine Eqs. (3.1) and (3.2). Since fluxes whose orders of magnitude are less than the proton flux $n_{0} V$ have been neglected, Eq. (3.1) is expanded to first order in $u \ll 1$ before combining. The result is

$$
J_{S}(\cos \alpha)=\frac{n_{0} v_{t}}{2 \sqrt{\pi}}\left[1-\sqrt{\pi} u \cos \alpha+o\left(u^{2}\right)\right]
$$

where $J_{s}=J_{-}-J_{+} \cdot$ Typically, $u \sim 0.2$ and therefore the dependence upon $\cos \alpha$ is weak. To lowest order in the small parameter $\operatorname{ucos} \alpha \ll 1$, the solar wind flux is given by the random electron flux. Also, note that $J_{s}$ is insensitive to variations in the bulk velocity $V$, and in the electron and proton temperatures. Finally, we emphasize that Eq. (3.3) is valid only if the lunar magnetic field is small enough to validate the assumption of a Maxwellian electron distribution for the incoming electrons near the lunar surface. 


\section{PHOTOELECTRON FLUX}

The average number of electrons with energy between $\varepsilon$ and $\varepsilon+d \varepsilon$ emitted by a photon of energy $E$ is denoted by

$$
\mathrm{n}_{\mathrm{E}}(\varepsilon) \mathrm{d} \varepsilon=\gamma(E) \mathrm{f}_{\mathrm{E}}(\varepsilon) \mathrm{d} \varepsilon
$$

Here $\gamma(E)$ is the quantum yield of the surface material and $f_{E}(\varepsilon)$ is the energy spectrum of photoemitted electrons, normalized so that

$$
\int_{0}^{\infty} d \varepsilon f_{E}(\varepsilon)=1 \text {. }
$$

$f_{E}(\varepsilon)$ embodies the fact that photons of a single energy $E$ produce electrons with a range of energies. It will be shown that this feature, which has been ignored in previous calculations of the lunar potential; serves to make $\mathrm{J}_{\mathrm{p}}(\Phi, \cos \theta)$, and thus the lunar potential, less sensitive to the small scale structures of the solar spectrum and the quantum yield than would be the case with monoenergetic emission of electrons by photons of a given energy.

If $g(E) d E$ is the flux of solar photons at the subsolar point with energy between $E$ and $E+d E$, the total photoemitted electron flux with energy between $\varepsilon$ and $\varepsilon+d \varepsilon$ at a point on the lunar surface whose radius vector from the center of the moon makes an angle $\theta$ with the moon-sun line, is

$$
\cos \theta d \varepsilon \int_{0}^{\infty} d E g(E) \gamma(E) f_{E}(\varepsilon) \text {. }
$$

Since the solar wind and solar photon flux directions are nearly parallel, $\cos \alpha$ may be replaced by $\cos \theta$ in $\mathrm{Eq} .(3.3)$.

of the total flux of photoemitted electrons given by Eq. (4.2), only those electrons with sufficient energy to overcome the electrostatic potential barrier will escape from the moon and contribute to $\mathrm{J}_{\mathrm{p}}$. Thus $\mathrm{J}_{\mathrm{p}}$ is given by

$J_{p}(\Phi, \cos \theta)=\cos \theta \int_{e^{\Phi}}^{\infty} d \varepsilon n(\varepsilon, \Phi) \int_{0}^{\infty} d E g(E) \gamma(E) f_{E}(\varepsilon)$ 
where $\eta(\varepsilon, \Phi)$ is the fraction of electrons of energy $\varepsilon$ which are emitted with perpendicular kinetic energy $\varepsilon_{\perp}=1 / 2 \mathrm{mv}_{\perp}^{2}>\mathrm{e} \Phi$ and which therefore escape. Here $v_{1}$ is the electron velocity component normal to the surface. For isotropic emission of electrons, $\eta$ is given by

$$
\eta(\varepsilon, \Phi)=1-(e \Phi / \varepsilon)^{1 / 2}, \varepsilon>e \Phi \geq 0 .
$$

The energy spectrum $f_{E}(\varepsilon)$ is typically a broad function going to zero at $\varepsilon=0$ and $\varepsilon=E-W$, where $W$ is the work runction of the emitting surface (see Figure la). The integration over E in Eq. (4.3) adds together many such broad functions of $\varepsilon$ which overlap over a wide range of energies. This process averages over the fine structure of $\gamma(E) g(E)$, resulting in a smooth function of $\varepsilon$ which reflects none of the small scale structure of $\gamma(E) g(E)$. This effect is shown schematically in Figure lb.

This smoothing process of the integral Eq. (4.3) is sufficiently effective to permit us to approximate the solar spectrum $g(E)$ by a smoothed function which averages over most of the emission peaks. Figure 2 shows the smoothed solar spectrum used in our calculations. The solar flux for quiet and active sun periods is indicated. Note that during solar minimum $g(E)$ rapidly decreases with increasing energy so that the most important contribution to $g(E)$ occurs for $E$ less than $\sim 100 \mathrm{eV}$. The process of photoemission in this energy range is well enough understood to allow for reasonable approximations to the shape of $\gamma(E)$ and $f_{E}(\varepsilon)$. We therefore restrict our attention to the case of solar minimum. A discussion of the possible effects of solar activity on the electrostatic potential is presented in the following section.

As the photon energy is increased above the work function $W$, typically about 5 volts, $\gamma(E)$ increases rapidly and reaches a plateau $\gamma_{\sim}^{\sim} \gamma_{0}$ at $\mathrm{E}_{\sim} \mathrm{E}_{1}$, and is reasonably constant until $\mathrm{E}_{\sim} \mathrm{E}_{2}$ (something like 30 or $40 \mathrm{eV}$ ), where $\gamma(E)$ begins to slowly decrease. At $\mathrm{E} \sim 80 \mathrm{eV}$, the threshold for exciting core electrons into the continuum, $\gamma(E)$ can be expected on theoretical grounds to rise again. A significant amount of structure may be superimposed on this gross behavior, but according to the arguments presented above, this structure is unimportant in computing $\mathrm{J}_{\mathrm{p}}(\Phi, \cos \theta)$. 
approximated by

These considerations suggest that $\gamma(E)$ may be

$$
\gamma(E)=\gamma_{0} H(E-W)
$$

where $H(x)$ is the unit step function.

It is apparent that any broad function of width $\Delta \varepsilon \sim(E-W)$ is an adequate representation of $f_{E}(\varepsilon)$. We choose

$$
f_{E}(\varepsilon)=\frac{2}{(E-W)} \sin ^{2}\left(\frac{\pi \varepsilon}{E-W}\right) H(-\varepsilon+E-W)
$$

which is normalized to unity with respect to integration over $\varepsilon$, and vanishes at $\varepsilon=0$ and $\varepsilon=E-W$, as required.

By substituting Eqs. (4.4)-(4.6) together with the smoothed solar spectrum $g(E)$ shown in Figure 2 into Eq. (4.3) and performing the integrations, we determine $J_{p}(\Phi, \cos \theta)$. The result depends upon two parameters, $\gamma_{0}$ and $W$, which characterize the photoemmisive properties of the lunar surface, and which are not known. For insulators and semiconductors $\gamma_{0}$ is typically between $10^{-3}$ and $10^{-1}$, and $w$ lies between 4 and 6 volts (see e.g., Apker, 1965; Maurer, 1966 and references listed there). It is plausible to expect that these ranges include the values of $\gamma_{0}$ and $W$ appropriate for the moon.

From Eq. (4.3) and the model Eq. (4.5) for $\gamma$ it is seen that $J_{p}$ is proportional to $\gamma_{0} \cos \theta$. It is therefore only necessary to compute the function $G(\Phi ; W)$, which represents the photoelectron flux at the subsolar point for unit quantum yield, defined by

$$
G(\Phi ; W)=\frac{J_{p}(\Phi, \cos \theta)}{\gamma_{0} \cos \theta} .
$$

The results are shown in Figure 3 .

5. POTENTIAL DISTRIBUTION

The electrostatic potential distribution $\Phi(\cos \theta)$ on the sunlit lunar surface is determined from the solution of the flux balance equation 
BELLCOMM, INC. $\quad-9-$

$$
\mathrm{J}_{\mathrm{p}}(\Phi, \cos \theta)=\mathrm{J}_{\mathrm{S}}(\Phi, \cos \theta)
$$

By using Eqs. (4.7) and (3.3) this becomes

$$
G(\Phi ; W)=\frac{n_{0} v_{t}}{2 \sqrt{\pi} \gamma_{0} \cos \theta}[1-\sqrt{\pi} u \cos \theta] .
$$

The potential distribution computed from Eq. (5.2) and Figure 3 depends upon three parameters $w, B=n_{0} v_{t} / \gamma_{0}$, and $u$, which characterize the solar wind and the lunar surface. Recall that the form of $G(\Phi ; W)$ shown in Figure 3 is valid only during solar minimum. From Eq. (5.2) and Figure 3 it is seen that, at a fixed surface point, the calculated electrostatic potential decreases monotonically with increasing $W$ or $\beta$. Since $u<<1$, the variation of $\Phi$ with $u$ is small, and we do not display this parametric dependence.

The potential distribution is shown in Figure 4 for a fixed value of $u$. The electrostatic potential at the subsolar point for median values of the plausible range of parameters is a few volts. An order of magnitude change in the parameter $\beta=n_{0} v_{t} / \gamma_{0}$ causes the potential to vary by about a factor of 2. Furthermore, the expected range for the work function limits the variation of potential with $W$ to less than a factor of 2. Finally, note that the condition $|\mathrm{e} \Phi|<<1 / 2 \mathrm{MV}^{2}$, assumed in section 3 , is satisfied for all reasonable values of the parameters.

The potential has a maximum at the subsolar point and decreases toward the limb. The solution given here is not valid over the entire sunlit hemisphere, but fails near the limb, as is evidenced by Eq. (5.2). This can be understood by recalling that, in computing the solar wind flux $\mathrm{J}_{\mathbf{s}}$, we relied on the existence of the ordering $J_{p}(0, \cos \theta)>>J_{s}(\Phi, \cos \theta), i . e .$, at a point on the surface the flux of emitted photoelectrons is much greater than the flux that can be drawn from the solar wind. In this case the calculation of the solar wind flux presented in Section 3 is appropriate. For all plausible values of $\beta$ and $\mathrm{W}$, it is seen from Eq. (5.2) and Figure 3 that, for some range of $\cos \theta$, the condition $\mathrm{J}_{\mathrm{p}}(0, \cos \theta)>>\mathrm{J}_{\mathrm{s}}(\Phi, \cos \theta)$ is satisfied, and our assumption is justified. However, since the solar 
BELLCOMM, INC.

photon flux normal to the sunlit surface has a $\cos \theta$ dependence, this ordering must fail as $\cos \theta \rightarrow 0$. In particular, Eq. (3.3) for $J_{s}$ becomes incorrect when $J_{p}$ is reduced to the point where $J_{p}(0, \cos \theta) \sim J_{s} \cdot$ This occurs for

$$
\cos \theta \sim \frac{\beta}{2 \sqrt{\pi} G(\Phi=0 ; W)}
$$

or

$$
\theta \sim \frac{\pi}{2}-\left[\frac{\beta}{\sqrt{\pi} G(\Phi=0 ; W)}\right]^{1 / 2}, \frac{\pi}{2}-\theta<<1 .
$$

Values of $G(\Phi=0 ; W)$ are presented in Table 1. As an illustration, if $W=5$ volts and $\beta=10^{11}\left(\mathrm{~cm}^{2}-\mathrm{sec}\right)^{-1}$, the solution breaks down when $\pi / 2-\theta \sim 10^{-2}$.

\section{TABLE 1}

Variation of $G(\Phi=0 ; W)$ with Work Function

\begin{tabular}{|l|c|c|c|c|}
\hline$W($ volts $)$ & 4 & 5 & 6 & 7 \\
\hline$G(\Phi=0 ; W)\left(\mathrm{cm}^{2}-\mathrm{sec}\right)^{-1}$ & $1 \times 10^{16}$ & $1 \times 10^{15}$ & $1 \times 10^{13}$ & $3 \times 10^{11}$ \\
\hline
\end{tabular}

As previously mentioned, these calculations were performed using the solar spectrum appropriate during solar minimum, although the formalism presented here is valid for the active sun case. It is impossible at present to predict the lunar potential during solar maximum due to a lack of photoemission data at photon energies above $\sim 100$ volts. As illustrated in Figure 2, the solar photon flux is significantly increased in the ultraviolet during solar maximum. This is also the case during flares. The variation of lunar potential with solar activity depends upon the magnitude of the quantum yield $\gamma(E)$ and the shape of the photoelectron distribution function $f_{E}(\varepsilon)$ for the energy range in which this enhancement occurs. The electrostatic potential varies significantly with solar activity only if the enhanced portion of the solar 
spectrum produces photoelectrons with energy greater than our computed electrostatic potential energy and with a flux greater than or comparable to $\mathrm{J}_{\mathbf{s}}$.

\section{CONCLUSIONS}

The electrostatic potential of the sunlit lunar surface during solar minimum is a maximum at the subsolar point and monotonically decreases toward the limb. For a lunar surface work function of +5 volts and a quantum yield of .01 , the subsolar point potential is 3.0 volts for typical solar wind conditions. As the work function and quantum yield vary between the plausible limits of 4 to 6 volts and .001 to .1, respectively, the subsolar point potential varies between +0.6 and 10.2 volts.

These results are insensitive to variations in the solar wind parameters and quantum properties of the lunar surface, and to the small scale structure of the solar spectrum. Furthermore, the subsolar point potential is an order of magnitude lower. than has been previously calculated (Öpik and Singer, 1960; Öpik, 1962; Heffner, 1965), due to the fact that more accurate account is taken of the solar spectrum and the energies of photoemitted electrons. These results imply that the corrections to the energies of charged particles measured by the ALSEP Suprathermal Ion Detector Experiment, or by similar lunar surface probes, will be unimportant at energies much greater than a few electron volts. Finally, the calculation presented here is a prerequisite for theoretical studies of the electric field at the lunar surface.

$1014-J L B$
$1015-$ WDG

\section{Attachments \\ References \\ Appendix \\ Figure Titles \\ Figures $1-4$}

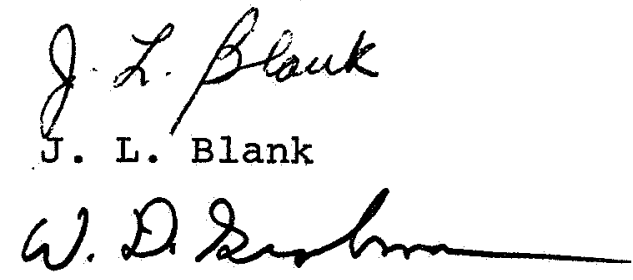

W. D. Grobman 
BELLCOMM, INC.

\author{
APPENDIX \\ Conduction Currents in the Photoelectron \\ Layer and the Lunar Crust
}

We first consider conduction parallel to the lunar surface by the photoelectrons. The electrical conductivity of the electrons is given by

$$
\sigma \sim \frac{\mathrm{Ne}^{2} \tau}{\mathrm{m}}
$$

where $\mathrm{N}$ is the photoelectron number density, and $\tau$ is the microscopic time scale for electron transport. The electron density is sufficiently low that collisions may be neglected. A representative photoelectron is emitted from the surface and, being unable to overcome the potential barrier, returns to the surface and is absorbed. Thus we identify $\tau$ as the time of flight of a typical electron.

We estimate $\tau$ from the electron equation of motion

$$
-\frac{e}{m} \frac{\partial \phi}{\partial z}=\frac{d^{2} z}{d t^{2}}
$$

where $\mathrm{z}$ is the height above the lunar surface and $\phi$ is the local value of the potential. For purposes of estimation, the actual potential gradient is replaced by $\Phi / \lambda_{D}$, where $\Phi$ is the surface potential, $\lambda_{D}=\left(\mathrm{kT}_{\mathrm{e}} / 4 \pi \mathrm{Ne} \mathrm{e}^{2}\right)^{1 / 2}$ is the photoelectron Debye length which represents the characteristic length scale for electrostatic shielding, and $\mathrm{T}_{e}$ is the photoelectron temperature. Then upon integrating twice and solving for the time of flight we obtain

$$
\tau \sim \sqrt{\frac{2}{\pi}} \frac{k T e}{e^{\Phi}} \frac{1}{{ }^{\omega_{p}}}
$$


where $\omega_{\mathrm{p}}=\left(4 \pi \mathrm{Ne}^{2} / \mathrm{m}\right)^{1 / 2}$ is the plasma frequency, and we have taken $d z(t=0) / d t=\left(k T e^{/ 2 \pi m}\right)^{1 / 2}$ which is the average vertical velocity for a Maxwellian distribution. Note that $t$ is of the order of the plasma period, which is just the time required for a thermal electron to travel a distance equal to the Debye length.

The photoelectron conductivity is then given by

$$
\sigma \sim \frac{\omega_{p}}{(2 \pi)^{3 / 2}} \frac{k T_{e}}{e^{\Phi}}
$$

The electron flux parallel to the surface is estimated from Ohm's law

$$
J_{11} \sim \frac{1}{\mathrm{e}} \sigma E_{11}
$$

Estimating $E_{\mid 1} \sim \Delta \Phi / R$, where $\Delta \Phi$ is the potential difference between the subsolar point and the limb, and $R$ is the lunar radius, and assuming $\Delta \Phi / \Phi \sim 1$, we obtain

where $\langle\mathrm{v}\rangle=\left(\frac{2 \mathrm{kT}}{\mathrm{m}}\right)^{1 / 2}$ is the photoelectron thermal speed. Thus the flux is essentially the thermal flux reduced by the factor $\lambda_{D} / R$. This factor is a geometrical one and may be understood in terms of the cross sectional areas through which the various fluxes flow. The treatment of the photoelectric process, presented in Section 4,indicates that the photoelectron density, or thermal flux, is not high enough to compensate for this geometrical factor. In fact, for $\gamma_{0}=10^{-1}$ and $w=4$ volts, $J_{p}(\Phi=0)=10^{15}\left(\mathrm{~cm}^{2}-\mathrm{sec}\right)^{-1}$. Therefore $\mathrm{J}_{\|} / \mathrm{J}_{\mathrm{s}} \ll 1$ for all reasonable values of $W$ and $\gamma_{0}$, and photoelectron transport processes are negligible. 
The absence of an observed disturbance in the solar wind on the sunlit side of the moon indicates that the lunar crust has an electrical conductivity $\sigma_{\mathrm{C}}<10^{-5}(\Omega-\mathrm{m}){ }^{-1}$ (Colburn et al., 1967; Ness et al., 1967). This upper bound is compatible with typical conductivities for dry rocks or rocks containing frozen water. The flux density due to conduction of current through the moon is

$$
J_{C} \sim \frac{1}{e} \sigma_{C} \frac{\Delta \Phi}{R}
$$

For $J_{C}$ to be comparable with the solar wind flux, $\Delta \Phi$ must be at least $\sim 10^{3}$ volts. Assuming no pathological behavior exists on the back side of the moon which can give rise to such large potentials, the computed potential differences render this conduction flux negligible. 
BELLCOMM, INC.

\section{REFERENCES}

Anonymous, Solar electromagnetic radiation, NASA SP-8005, 1965.

Apker, L., Photoemission, in McGraw-Hill Encyclopedia of Science and Technology, Vol. 10, p. 152, McGraw Hill, New York, 1965.

Chen, F. F., Electric probes, in Plasma Diagnostic Techniques, edited by R. H. Huddlestone and S. L. Leonard, P. 113, Academic Press, New York, 1965.

Colburn, D. S., R. G. Currie, J. D. Milhalov, and C. P. Sonnett, Diamagnetic solar-wind cavity discovered behind moon, Science, 158, 1040, 1967.

Edwards, W. F., and L. B. Borst, Possible sources of a lunar atmosphere, Science, 127, 325, 1958.

Hagstrum, H. D., Theory of Auger ejection of electrons from metals by ions, phys. Rev., 96, 336, 1954 .

Hagstrum, H. D., Auger electron ejection from germanium and silicon by noble gas ions, Phys. Rev., 119, 940, 1960.

Heffner, H., The potential and electric field at the surface of the moon, report TG-7 of the "Tycho" Study Group, University of Minnesota, 1965.

Hundhausen, A. J., J. R. Asbridge, S. J. Bame, H. E. Gilbert, and I. B. Strong, Vela 3 satellite observations of solar wind ions: A preliminary report, J. Geophys. Res., $\underline{72}$, 87,1967 .

Maurer, R. J., Photoelectric effect, in Handbook of Physics, edited by E. U. Condon and H. Odishaw, p. 8-66, McGrawHill, New York, 1966.

Montgomery, M. D., S. J. Bame, and A. J. Hundhausen, Solar wind electrons: Vela 4 measurements, J. Geophys. Res., 73, 4999, 1968 .

Ness, N. F., K. W. Behannon, C. S. Scearse, and S. C. Cantarano, Early results from the magnetic field experiment on lunar Explorer 35, J. Geophys. Res., 72, 5769, 1967. 


\section{BELLCOMM, INC.}

References (Cont'd)

Opik, E. J., The lunar atmosphere, Planet. Space Sci., 9, 221, 1962 .

Öpik, E. J., and S. F. Singer, Escape of gases from the moon, J. Geophys. Res., 65, 3065, 1960 .

Weil, H., and M. L. Barasch, A theoretical lunar ionosphere, Icarus, $1,346,1963$. 
BELLCOMM, INC.

FIGURE TITLES

Fig. 1. a) Schematic representation of the energy spectrum $f_{E}(\varepsilon)$ of electrons of energy $\varepsilon$ emitted by photons of energy $\mathrm{E}$.

b) Schematic illustration of the fact that the integral $\int \mathrm{dE} \gamma(E) g(E) f_{E}(\varepsilon)$ is a smooth function of $\varepsilon$ even for "noisy" functions $\gamma(E)$ and $g(E)$.

Fig. 2. Smoothed solar spectrum used in calculating the integral (7). This curve is taken from Anonymous [1965]. Below about $1400 \AA$, the true spectrum consists mostly of sharp emission lines, and the above curve is obtained by smoothing over $50 \AA$ intervals.

Fig. 3. Plot of $G(\Phi ; W)$, the photoelectron flux at the subsolar point for unit quantum yield, during solar minimum as a function of potential $\Phi$, for three different values of the work function $W$.

Fig. 4. Electrostatic potential distribution of the sunlit lunar hemisphere during solar minimum. The parametric dependence upon $W$ and $\beta=n_{0} v_{t} / \gamma_{0}$ is shown for the fixed value of $u$ corresponding to $V=400 \mathrm{~km} / \mathrm{sec}$, $T=10^{5} \mathrm{~K}$. For a solar wind density of 5 particles/ $\mathrm{cm}^{3}, \beta=10^{11}\left(\mathrm{~cm}^{2}-\mathrm{sec}\right)^{-1}$ corresponds to a quantum yield $\gamma_{0}=8.7 \times 10^{-3}$. 

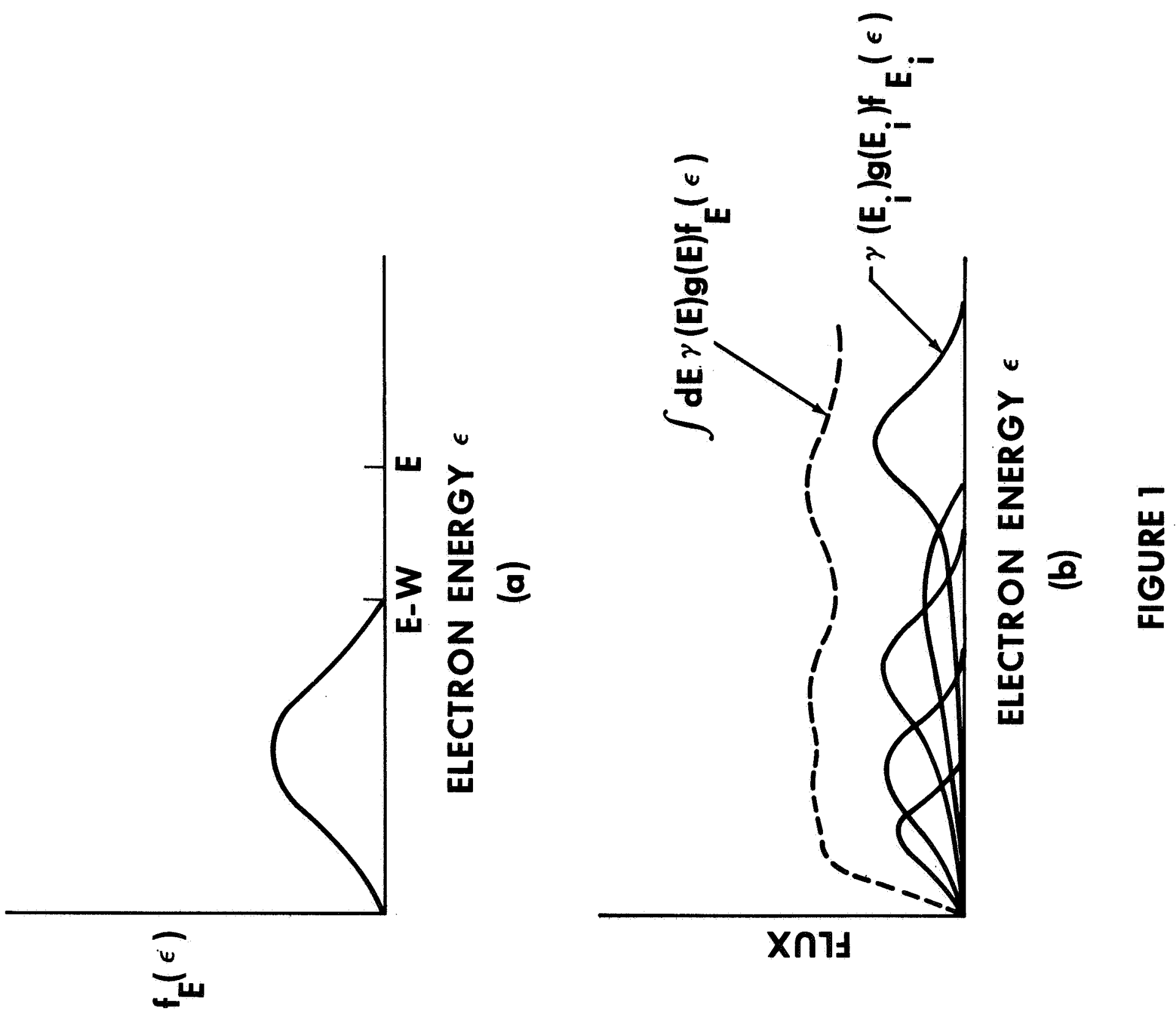


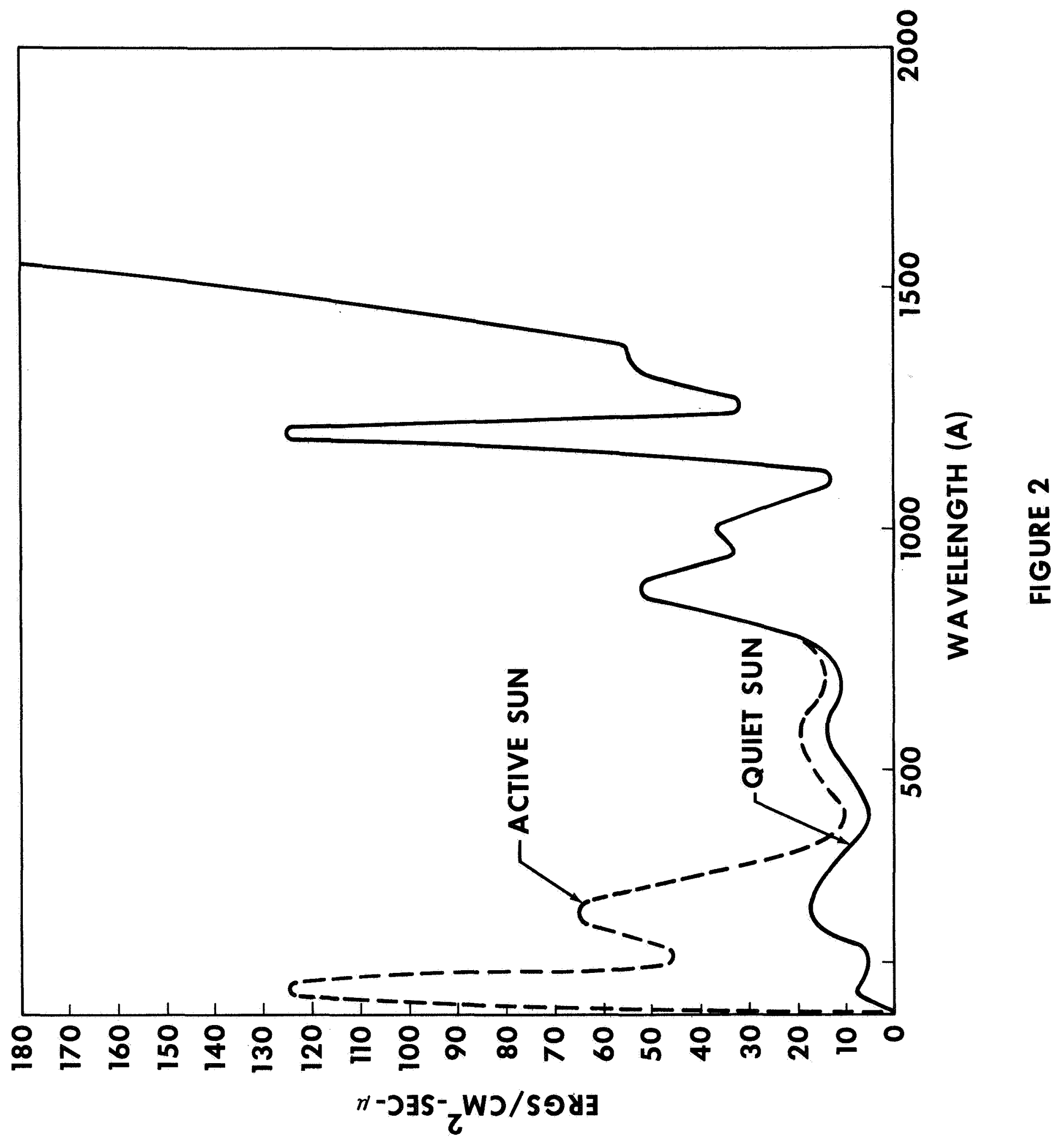




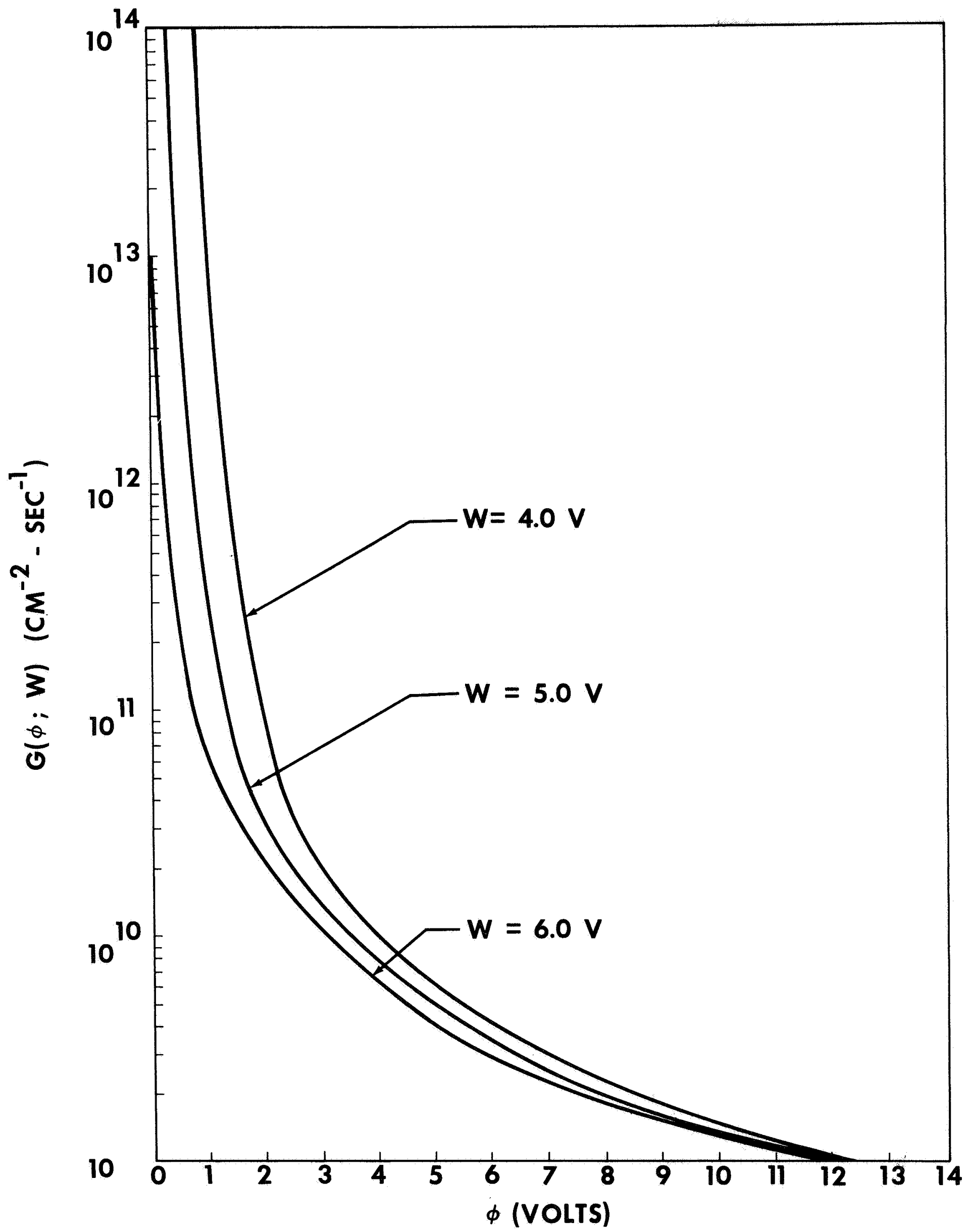

FIGURE 3 


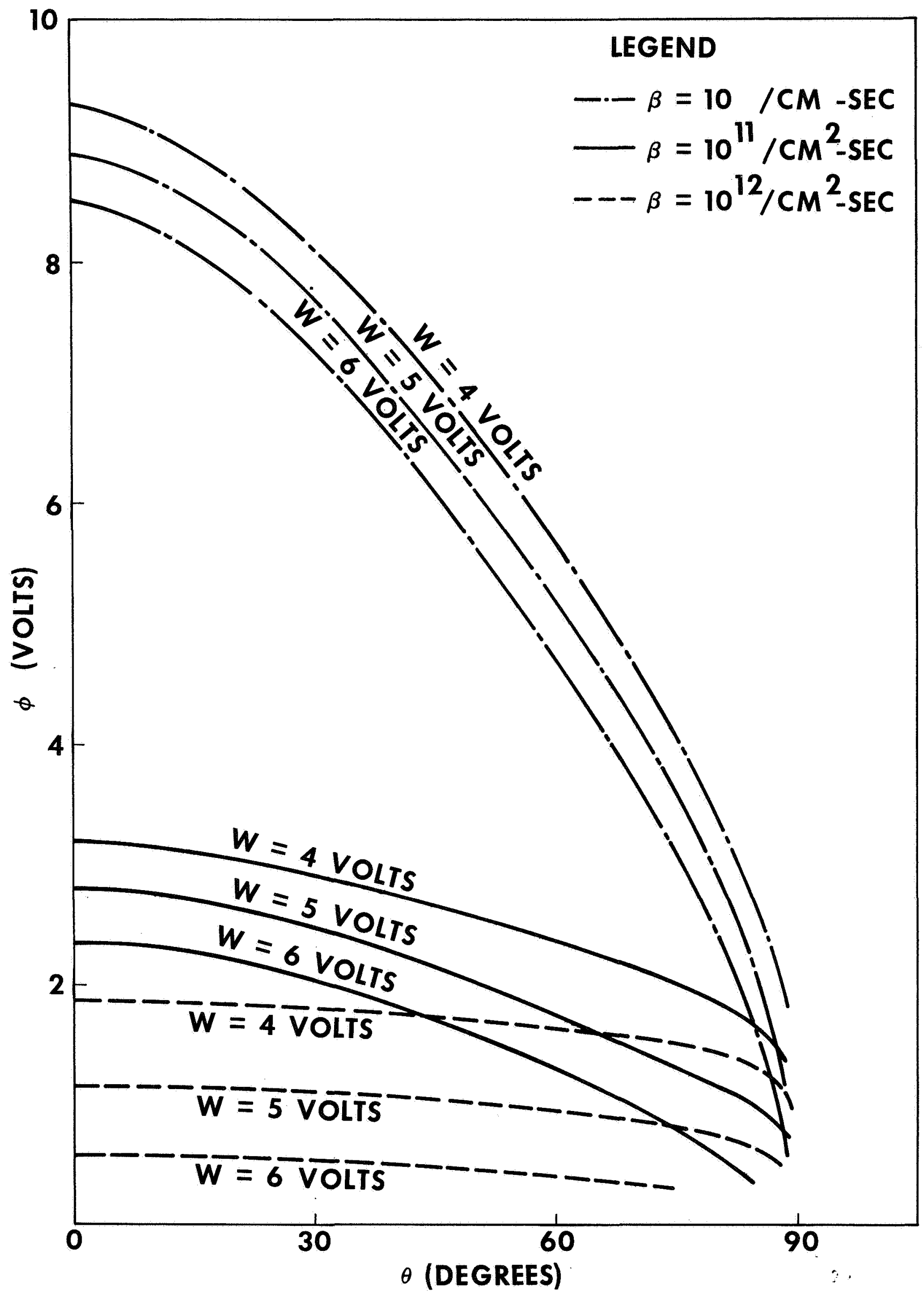

FIGURE 4 
BELLCOMM, INC.

Distribution List

NASA Headquarters

Messrs. W. O. Armstrong/MTX

C. J. Donlan/MD-T

E. W. Hall/MTG

H. $\mathrm{Hall} / \mathrm{MTX}$

T. A. Keegan $/ M A-2$

R. L. Lohman/MTY

D. R. Lord/MTD

S. C. Phillips/MA

L. Roberts/OART-M

A. D. Schnyer/MTV

M. G. Waugh/MTP

J. W. Wild/MTE

D. U. Wise/MAL

NASA Hqts. Library/USS-10

Ames Research Center

W. I. Linlor/SSE

C. P. Sonett/SS

Electronic Research Center

E. H. Walker/KIA

Goddard Space Flight Center

J. P. Heppner $/ 612$

N. F. Ness $/ 612$

J. A. O'Keefe/640

Manned Spacecraft Center

W. N. Hess/TA

O. K. Garriott/CB

R. H. Manka/TG5

J. L. Modisette/RG

Cornell University

T. Gold

Massachusetts Institute of Technology

H. S. Bridge
National Center for Atmospheric Research

E. W. Walbridge

Princeton University

J. J. Hopfield

R. A. Phinney

Rice University

H. Anderson

A. J. Dessler

J. Freeman

F. C. Michael

Southwest Center for Advanced Studies

F. S. Johnson/DASS

Stanford Electronics Laboratories

W. E. Spicer

Stanford University

H. Heffner

United States Department of Interior

S. F. Singer

University of California at Berkeley

F. S. Mozer

University of Maryland

E. J. Öpik

University of Southern California

G. Weissler 
BELLCOMM, INC.

Distribution List (Continued)

Bellcomm, Inc.

Messrs. F. G. Allen
G. M. Anderson
D. J. Belz
A. P. Boysen, Jr.
D. A. Chisholm
C. I. Davis
D. A. DeGraaf
D. R. Hagner
N. W. Hinners
W. W. Hough
B. T. Howard
D. B. James
J. Kranton
H. S. London
K. E. Martersteck
J. Z. Menard
G. T. Orrok
I. M. Ross
W. B. Thompson
J. W. Timko
A. R. Vernon
R. I. Wagner
J. E. Waldo
Members Division 101
All 15
All Members Department 2015
Department 1024 File
Librtral Files
Fily 